\title{
Holomorphic structures in hydrodynamical models of nearly geostrophic flow
}

\author{
By V. N. Roubtsov And I. Roulstone \\ ${ }^{1}$ Département de Mathématiques, Université d'Angers, \\ 2 Blvd Lavoisier, 49045 Angers, France and \\ ITEP, 117259, Moscow, Russia (volodya@tonton.univ-angers.fr) \\ ${ }^{2}$ Met Office, Joint Centre for Mesoscale Meteorology, University of Reading, \\ Earley Gate, Reading RG6 6BB, UK (iroulstone@meto.gov.uk)
}

\section{Received 15 February 2000; revised 30 November 2000; accepted 4 December 2000}

We study complex structures arising in Hamiltonian models of nearly geostrophic flows in hydrodynamics. In many of these models an elliptic Monge-Ampère equation defines the relationship between a 'balanced' velocity field, defined by a constraint in the Hamiltonian formalism, and the materially conserved potential vorticity. Elliptic Monge-Ampère operators define an almost-complex structure, and in this paper we show that a natural extension of the so-called geostrophic momentum transformation of semi-geostrophic theory, which has a special importance in theoretical meteorology, defines Kähler and special Kähler structures on phase space. Furthermore, analogues of the 'geostrophic momentum coordinates' are shown to be special Lagrangian coordinates under conditions which depend upon the physical approximations under consideration. Certain duality properties of the operators are studied within the framework of the Kähler geometry.

Keywords: holomorphic function; Hamiltonian structure; hydrodynamics;

Kähler geometry; special Lagrangian coordinates; Monge-A mpére equations

\section{Introduction}

The very complicated flow of both the atmosphere and oceans is believed to be described accurately by the classical Navier-Stokes-based equations of fluid motion. However, in the asymptotic regimes (parametrized by rotation and stratification) that are most relevant for weather and climate forecasting, it can be shown that the solutions of the fluid equations stay close over finite, but useful, time intervals to the solutions of much simpler dynamical systems. These approximate models seek to describe flows in which there is a dominant balance between the Coriolis, buoyancy and pressure-gradient forces on fluid particles. Such approximations to Newton's second law are commonly referred to as balanced models, and the so-called quasigeostrophic and semi-geostrophic theories are examples of such models.

Salmon $(1983,1985,1988)$ pioneered the systematic derivation of balanced models within the framework of Hamilton's principle. The rationale is to make approximations to the Lagrangian without disturbing the symmetry properties of the functional, thereby ensuring that the resulting model retains approximations to the conservation 
laws of the Euler equations. In particular, the particle relabelling symmetry corresponds, within the model approximations, to the fundamental meteorological cum oceanographic fluid-dynamical principle of conservation of 'potential vorticity'.

The semi-geostrophic model has a particular significance in dynamical meteorology. The reason is that semi-geostrophy has certain useful and elegant mathematical properties, in particular Hamiltonian structure, Legendre duality, and the convexity of certain potential functions (Chynoweth \& Sewell 1989, 1991; Roulstone \& Norbury 1994; Roulstone \& Sewell 1997). These permit both robust numerical integrationeven in cases where frontal discontinuities form - and a rather complete knowledge of mathematical properties, such as existence and uniqueness of solutions (Cullen \& Purser 1984; Purser \& Cullen 1987; Cullen et al. 1991). Subsequently, within a class of balanced models described by McIntyre \& Roulstone (1996), quaternionic and Kähler structure has been identified. This structure is a consequence of the relationship between the potential vorticity and a geopotential height function which is governed by a Monge-Ampère equation (Roubtsov \& Roulstone 1997). In light of this discovery, McIntyre \& Roulstone (2001) address the issue of whether higherorder corrections to semi-geostrophy may be constructed while retaining some of the mathematical features that facilitate the integration of the equations both analytically and numerically. This issue is of practical importance given the diversity of balanced models used to study the various regimes of geophysical flows (Allen et al. 1990; McWilliams \& Gent 1980), and the need for efficient, accurate integration schemes for such models.

In the spirit of these recent developments, the purpose of this paper is to demonstrate the existence of Kähler, special Lagrangian and special Kähler geometry (see $\S \S 3,4 a-c)$ in the models described by McIntyre \& Roulstone (1996). In turn, this facilitates the study of certain duality properties (see $\S \S 4 c, d$ ). Hitherto, duality has only been studied within the context of the semi-geostrophic equations, which do not possess Kähler structure. We shall use the shallow-water equations as a paradigm for the equations of meteorology and oceanography, because the absence of an explicit form of momentum balance in the vertical facilitates a clear exposition of the transformations of the horizontal components of the configuration and velocity fields.

\section{Shallow-water theory and balanced dynamics}

The motion of a typical particle in shallow-water theory can be described by expressing the current Cartesian horizontal coordinates

$$
x=x(a, b, t), \quad y=y(a, b, t)
$$

as functions, on the right of (2.1), of the Lagrangian particle labels $a, b$ and the time $t$. The incompressibility hypothesis requires the current depth, or height, $h$ to be a function $h(a, b, t)$ with the property

$$
\frac{h(a, b, 0)}{h(a, b, t)}=\frac{\partial(x, y)}{\partial(a, b)},
$$

where the Jacobian on the right-hand side is that of the mapping (2.1). The time derivative of (2.2) following a particle gives the differential equation of continuity.

The equations of horizontal momentum balance for flows over a bed which is rotating with position-dependent Coriolis parameter $f(y)$ (where the $y$-coordinate 
denotes north) are

$$
\ddot{x}+g \frac{\partial h}{\partial x}-\dot{y} f=0, \quad \ddot{y}+g \frac{\partial h}{\partial y}+\dot{x} f=0 .
$$

Here $g$ is a given constant representing the combined effect of the acceleration due to gravity and a centrifugal component due to the Earth's rotation, and the superposed dot denotes the time derivative following a particle.

Another important kinematical concept is the so-called potential vorticity defined by

$$
\xi=\frac{1}{h}\left(\frac{\partial \dot{y}}{\partial x}-\frac{\partial \dot{x}}{\partial y}+f\right),
$$

which is conserved on particles. Common choices of the Coriolis parameter are $f=$ const. or $\beta y$, as approximations to $2 \Omega \sin \phi$, depending on purpose. Here $\beta$ and $\Omega$ are constants (related to the spin of the Earth), and $\phi$ is latitude. (Note, to avoid confusion, that $\Omega$ will be used to denote a symplectic structure later in the paper.)

The semi-geostrophic approximation to equations (2.3), in the case when $f$ is a constant, is the replacement of the true acceleration by the time derivative of another vector,

$$
u_{g}=-\frac{g}{f} \frac{\partial h}{\partial y}, \quad v_{g}=\frac{g}{f} \frac{\partial h}{\partial x},
$$

following the particle. This vector is a notional velocity, called the geostrophic velocity. The semi-geostrophic approximation seeks to find motions satisfying

$$
\dot{u}_{g}+g \frac{\partial h}{\partial x}-\dot{y} f=0, \quad \dot{v}_{g}+g \frac{\partial h}{\partial y}+\dot{x} f=0,
$$

together with the continuity equation obtained from (2.2). Associated with these equations, the potential vorticity

$$
\Xi=\frac{1}{h}\left[f+\frac{\partial v_{g}}{\partial x}-\frac{\partial u_{g}}{\partial y}+\frac{1}{f} \frac{\partial\left(u_{g}, v_{g}\right)}{\partial(x, y)}\right],
$$

is conserved.

Following Hoskins (1975) we introduce a transformation of coordinates:

$$
X=x+\frac{g}{f^{2}} \frac{\partial h}{\partial x}, \quad Y=y+\frac{g}{f^{2}} \frac{\partial h}{\partial y} .
$$

For a thorough discussion of this transformation, including its Legendre and contact properties, see Roulstone \& Sewell (1997). The coordinates $(X, Y)$ are called geostrophic coordinates, because, when $f$ is a constant, $\dot{X}=u_{g}, \dot{Y}=v_{g}$. Motivated by the issue of the dependence of the Coriolis parameter, $f$, on latitude, Salmon (1985) studied certain generalized semi-geostrophic equations with pseudoHamiltonian form in $(X, Y)$-space, namely

$$
\dot{X}=-\frac{1}{f(X, Y)} \frac{\partial \Psi}{\partial Y}, \quad \dot{Y}=\frac{1}{f(X, Y)} \frac{\partial \Psi}{\partial X},
$$

where

$$
\Psi(X, Y, t)=\frac{1}{2}\left(u_{g}^{2}+v_{g}^{2}\right)+g h .
$$


The results of Salmon (1985) are also of interest when $f$ is independent of position (in which case (2.9) can easily be written in canonical form) and henceforth, in this paper, we consider $f$ to be a given constant. In the coordinates (2.8) the potential vorticity takes the Jacobian form (noting (2.5)):

$$
\Xi=\frac{f}{h} \frac{\partial(X, Y)}{\partial(x, y)}=\frac{1}{h}\left[f+\frac{\partial v_{g}}{\partial x}-\frac{\partial u_{g}}{\partial y}+\frac{1}{f} \frac{\partial\left(u_{g}, v_{g}\right)}{\partial(x, y)}\right] .
$$

The definition (2.11) can be rewritten as

$$
\Xi=\frac{g f}{\left(H-\frac{1}{2} f\left(x^{2}+y^{2}\right)\right)}\left|\begin{array}{cc}
\frac{\partial^{2} H}{\partial x^{2}} & \frac{\partial^{2} H}{\partial x \partial y} \\
\frac{\partial^{2} H}{\partial y \partial x} & \frac{\partial^{2} H}{\partial y^{2}}
\end{array}\right|,
$$

where $H=\frac{1}{2} f\left(x^{2}+y^{2}\right)+g h$. This is the starting point for a Monge-Ampère type of equation in which $H(x, y, t)$ is the unknown, to be found in conjunction with suitable boundary conditions. Roubtsov \& Roulstone (1997) show how the Monge-Ampère equation (2.12) can be expressed in terms of a 2-form restricted to the graph of $h$.

McIntyre \& Roulstone (1996) studied a family of balanced models, all of which possess a 'Monge-Ampère/stream function' relationship, like the semi-geostrophic equations above, between the potential vorticity and the depth $h$. These balanced models are termed 'near-local' because they can be derived using the framework of constrained Hamiltonian systems (Salmon 1988; McIntyre \& Roulstone 1996) in which the constraints depend on the local value of the depth function and a finite number of its derivatives with respect to $x$ and $y$. No non-local constraints are involved. Canonical coordinates, which should be considered as analogues of (2.8), were discovered together with an associated complex structure, and this was formulated in terms of quaternions by Roubtsov \& Roulstone (1997).

\section{Complex and Kähler structures}

Following McIntyre \& Roulstone (1996), Roubtsov \& Roulstone (1997) introduce the following notation for canonical coordinates for near-local balanced models

$$
X=x+\mathrm{i} c q+a p, \quad Y=y-\mathrm{i} c p+a q,
$$

where $a, c \in \mathbb{R}, \mathrm{i}=\sqrt{-1}$ and $(p, q)=(g \partial h / \partial x, g \partial h / \partial y)$. This particular choice (noting the presence of the minus sign in the definition of $Y$ ) was motivated by the fact that McIntyre \& Roulstone (1996) showed that the materially conserved potential vorticity, $\Xi$, can always be expressed in Jacobian form (cf. (2.11) and $(2.12))$ :

$$
\Xi=\frac{f}{h} \frac{\partial(X, Y)}{\partial(x, y)} .
$$

The parameters $a$ and $c$ determine the form of the potential vorticity (and vice versa) and the choice is fixed by the balanced model under consideration. However, we shall find $(\S 4 b)$ that the expression for the coordinates (3.1) is subject to certain restrictions if particular complex structures are required. 
Another reason for making the choice (3.1) concerns the relationship between this set of coordinates and those defined in (2.8). In particular, if we define $\hat{z}=x+\mathrm{i} c q$, $\hat{w}=y-\mathrm{i} c p$ on a two-dimensional complex manifold, then there exists a hyper-Kähler triple of 2 -forms

$$
\begin{aligned}
& \hat{\omega}_{I}=\operatorname{Re}(\mathrm{d} \hat{z} \wedge \mathrm{d} \hat{w})=\mathrm{d} x \wedge \mathrm{d} y-c^{2} \mathrm{~d} p \wedge \mathrm{d} q, \\
& \hat{\omega}_{J}=\frac{1}{2} \mathrm{i}(\mathrm{d} \hat{z} \wedge \mathrm{d} \overline{\hat{z}}+\mathrm{d} \hat{w} \wedge \mathrm{d} \overline{\hat{w}})=c(\mathrm{~d} x \wedge \mathrm{d} q+\mathrm{d} p \wedge \mathrm{d} y), \\
& \hat{\omega}_{K}=\operatorname{Im}(\mathrm{d} \hat{z} \wedge \mathrm{d} \hat{w})=-c(\mathrm{~d} x \wedge \mathrm{d} p+\mathrm{d} y \wedge \mathrm{d} q)=-c \Omega,
\end{aligned}
$$

where $\Omega$ is the canonical 2-form. The first two 2-forms, (3.3) and (3.4), represent Legendre-conjugate elliptic Monge-Ampère operators (see Lychagin et al. (1993) for the general theory). The transformation $(\hat{z}, \hat{w}) \mapsto(X, Y)$, implicit in $(3.1)$, has the following action on the hyper-Kähler triple (i.e. $\mathrm{d} \hat{z} \wedge \mathrm{d} \hat{w} \mapsto \mathrm{d} X \wedge \mathrm{d} Y$, etc.):

$$
\begin{aligned}
\hat{\omega}_{I} & \mapsto \mathrm{d} x \wedge \mathrm{d} y+a(\mathrm{~d} x \wedge \mathrm{d} q+\mathrm{d} p \wedge \mathrm{d} y)+\left(a^{2}-c^{2}\right) \mathrm{d} p \wedge \mathrm{d} q, \\
\hat{\omega}_{J} & \mapsto c(\mathrm{~d} x \wedge \mathrm{d} q+\mathrm{d} p \wedge \mathrm{d} y)+2 a c \mathrm{~d} p \wedge \mathrm{d} q, \\
\hat{\omega}_{K} & \mapsto \hat{\omega}_{K} \quad(=-c \Omega) .
\end{aligned}
$$

Once again, the first two 2-forms represent elliptic Monge-Ampère operators, i.e. the transformation (3.1) preserves the ellipticity, and for the choice $a=1 / f^{2}, c=0$ we recover (2.8), and the equations that follow, from (3.1). The elliptic operators discussed by McIntyre \& Roulstone (1996) correspond to different choices of $a$ and $c$ in $(3.6)$.

It is convenient for the purposes of this paper to introduce a new notation. Define

$$
\left.\begin{array}{rl}
Z \equiv x+\mathrm{i} c q+a p & (=X), \\
W \equiv y+\mathrm{i} c p+a q & (=\bar{Y}),
\end{array}\right\}
$$

and we shall show (see (3.14) and those that follow) that there exists a Kähler structure in this representation. The salient point is that we wish to place the emphasis on the complex structure rather than on the Jacobian relationship (3.2) between the potential vorticity, $\Xi$, and the 'geostrophic coordinates' $(X, Y)$.

From (3.9), one can readily show that

$$
\frac{\partial(Z, \bar{Z}, W, \bar{W})}{\partial(x, y, p, q)}=-4 c^{2}
$$

and we find

$$
\begin{gathered}
p=\frac{\mathrm{i}}{2 c}(\bar{W}-W), \quad q=-\frac{\mathrm{i}}{2 c}(Z-\bar{Z}), \\
x=\frac{1}{2}(Z+\bar{Z})-\frac{\mathrm{i} a}{2 c}(\bar{W}-W), \quad y=\frac{1}{2}(W+\bar{W})+\frac{\mathrm{i} a}{2 c}(Z-\bar{Z}) .
\end{gathered}
$$

It follows that

$$
\frac{\partial}{\partial Z}=\frac{1}{2}\left(\frac{\partial}{\partial x}+\frac{\mathrm{i} a}{c} \frac{\partial}{\partial y}-\frac{\mathrm{i}}{c} \frac{\partial}{\partial q}\right), \quad \frac{\partial}{\partial W}=\frac{1}{2}\left(\frac{\partial}{\partial y}+\frac{\mathrm{i} a}{c} \frac{\partial}{\partial x}-\frac{\mathrm{i}}{c} \frac{\partial}{\partial p}\right) .
$$

The canonical structure in the coordinates $(Z, W)$ is evidently a $(1,1)$-form,

$$
\Omega=\frac{\mathrm{i}}{2 c}(\mathrm{~d} Z \wedge \mathrm{d} \bar{W}+\mathrm{d} W \wedge \mathrm{d} \bar{Z})=\mathrm{d} x \wedge \mathrm{d} p+\mathrm{d} y \wedge \mathrm{d} q,
$$


and can be used to define a Kähler structure of the model defined by the particular choice of real parameters $a$ and $c$. A second argument (see $\S 4 b$ ) for adopting the coordinates $(Z, W)$ is that they can be considered as an example of special Lagrangian coordinates on an 'initial' phase space spanned by a set of coordinates $(z, w)$,

$$
z \equiv x+\mathrm{i} c q, \quad w \equiv y+\mathrm{i} c p,
$$

with deformed complex structure

$$
(z, w) \mapsto(Z, W),
$$

in accordance with the prescriptions for their existence given by Hitchin (1997) (see also Gross 1999). The 'momentum' part of the transformation $(p, q) \mapsto(\mathcal{P}, \mathcal{Q})$,

$$
\mathcal{P} \equiv a p+\mathrm{i} c q, \quad \mathcal{Q} \equiv \mathrm{i} c p+a q,
$$

has a diagonal real part and symmetric imaginary part, and hence the coordinates $(Z, W)$ obey, trivially, the prescriptions to be special Lagrangian. We recall that this property means that there is an integrable almost-complex structure on the phase space of the model, which induces a flat metric such that the cotangent components $\mathcal{P}, \mathcal{Q}$ (which are evidently Lagrangian) are also of 'minimal volume' with respect to this metric. These conditions are discussed in detail in theorem 5.2 in Gross (1999). This observation shows that the phase space of the near-local balanced models with the coordinates $(Z, W)$ can be considered as a Lagrangian fibration over the base manifold with local coordinates $(x, y)$. The Ricci-flat condition, under which the notion 'special Lagrangian' is defined, corresponds, for example, to the MongeAmpère equation (3.2) with $\Xi h=1$ (cf. (3.6) recalling that $a, c \in \mathbb{R}$ ). We shall return to this point in $\S 4 c$.

The holomorphic (2,0)-form $\mathrm{d} Z \wedge \mathrm{d} W$ is expressed in the original coordinates as

$$
\begin{aligned}
\mathrm{d} Z \wedge \mathrm{d} W=(\mathrm{d} z+a \mathrm{~d} p) \wedge(\mathrm{d} w+a \mathrm{~d} q) \\
=\mathrm{d} x \wedge \mathrm{d} y+a(\mathrm{~d} x \wedge \mathrm{d} q+\mathrm{d} p \wedge \mathrm{d} y) \\
\quad+\mathrm{i} c(\mathrm{~d} x \wedge \mathrm{d} p+\mathrm{d} q \wedge \mathrm{d} y)+\left(a^{2}+c^{2}\right) \mathrm{d} p \wedge \mathrm{d} q .
\end{aligned}
$$

We will see below that this is essentially the only possible choice of complex structure once we fix the elliptic Monge-Ampère operator for the balanced model. From the hyper-Kähler geometry point of view, the adoption of the definition (3.15) is nothing more than choosing to work with the canonical flat hyper-Kähler structure on $\mathbb{C}^{2}$ :

$$
\begin{aligned}
\omega_{I} & =\operatorname{Re}(\mathrm{d} z \wedge \mathrm{d} w), \\
\omega_{J} & =\frac{1}{2} \mathrm{i}(\mathrm{d} z \wedge \mathrm{d} \bar{z}+\mathrm{d} w \wedge \mathrm{d} \bar{w}), \\
\omega_{K} & =\operatorname{Im}(\mathrm{d} z \wedge \mathrm{d} w) .
\end{aligned}
$$

But we then find that under (3.16), which we represent as $\omega \mapsto \tilde{\omega}$, we have

$$
\begin{aligned}
\tilde{\omega}_{I} & =\mathrm{d} x \wedge \mathrm{d} y+a(\mathrm{~d} x \wedge \mathrm{d} q+\mathrm{d} p \wedge \mathrm{d} y)+\left(a^{2}+c^{2}\right) \mathrm{d} p \wedge \mathrm{d} q, \\
\tilde{\omega}_{J} & =c(\mathrm{~d} x \wedge \mathrm{d} q-\mathrm{d} p \wedge \mathrm{d} y) \quad\left(=\omega_{J}\right), \\
\tilde{\omega}_{K} & =c(\mathrm{~d} x \wedge \mathrm{d} p+\mathrm{d} q \wedge \mathrm{d} y),
\end{aligned}
$$

and now the triple represent hyperbolic Monge-Ampère operators. So we have lost ellipticity, a feature which is crucial to the physical application in mind. We establish the connection between elliptic operators, the choice of coordinates on $\mathbb{C}^{2}$, and the special Kähler structure, in $\S 4 c$. 


\section{Monge-Ampére operators and Kähler geometry}

(a) Further remarks on Kähler structure

There exists a (complex-valued) generating function

$$
S=\mathrm{i} c p q+\frac{1}{2} a\left(p^{2}+q^{2}\right)
$$

such that

$$
Z-x=\frac{\partial S}{\partial p}=\mathrm{i} c q+a p, \quad W-y=\frac{\partial S}{\partial q}=\mathrm{i} c p+a q .
$$

Let us consider a potential function $\Phi=\varphi+S$, where $\varphi \equiv g h$ and hence $p=$ $\partial \varphi / \partial x$ and $q=\partial \varphi / \partial y$. Direct verification, using (3.13), shows that the function $\Phi$ is holomorphic in the coordinates $(Z, W)$ :

$$
\frac{\partial \Phi}{\partial \bar{Z}}=0, \quad \frac{\partial \Phi}{\partial \bar{W}}=0
$$

and

$$
\frac{\partial \Phi}{\partial Z}=p, \quad \frac{\partial \Phi}{\partial W}=q
$$

From the derivatives of the potential function $\Phi$ we can construct the matrix

$$
\left\|\tau_{i j}\right\|=\left(\begin{array}{cc}
\frac{\partial^{2} \Phi}{\partial Z^{2}} & \frac{\partial^{2} \Phi}{\partial Z \partial W} \\
\frac{\partial^{2} \Phi}{\partial W \partial Z} & \frac{\partial^{2} \Phi}{\partial W^{2}}
\end{array}\right)=\left(\begin{array}{cc}
0 & -\frac{\mathrm{i}}{2 c} \\
-\frac{\mathrm{i}}{2 c} & 0
\end{array}\right)
$$

and a real function

$$
K=\frac{1}{2} \operatorname{Im}\left(\frac{\partial \Phi}{\partial Z} \bar{Z}+\frac{\partial \Phi}{\partial W} \bar{W}\right)=-c p q .
$$

We see that (4.5) is a Kähler potential: it is (up to the minus sign) the imaginary part of our holomorphic potential $\Phi$ and our canonical form (3.14) is

$$
\Omega=-2 \mathrm{i} \partial \bar{\partial} \mathrm{K}=-\mathrm{i} \operatorname{Im}\left(\tau_{i j}\right) \mathrm{d} u_{i} \wedge \mathrm{d} \bar{u}_{j},
$$

where $u_{1}=Z, u_{2}=W$. Our interest in the holomorphic function $\Phi$ stems from the analogy to $(2.10)$ when $c=0$, and we shall return to this issue in $\S 4 d$.

\section{(b) A link to Monge-Ampére operators}

Let $\Omega$ be the canonical symplectic form and $\omega$ be a non-degenerate 2 -form on $\mathbb{R}^{4}$ representing an elliptic Monge-Ampère operator (Lychagin 1979; Roubtsov \& Roulstone 1997). This means that we have an almost-complex structure on $\mathbb{R}^{4}$ whose integrability condition (vanishing of the Nijenhuis tensor) is equivalent to the condition that the form $\omega$, by a (conformal) symplectomorphism of $\mathbb{R}^{4}$, is reduced to a form with constant coefficients and its Pffafian is equal to 1 (Lychagin et al. 1993).

We can choose a holomorphic $(2,0)$-form corresponding to this complex structure in such a way that

$$
\mathrm{d} \tilde{Z} \wedge \mathrm{d} \tilde{W}=\omega-\mathrm{i} \Omega .
$$


Now we can address the following question: what freedom of choice is there in the special Lagrangian coordinates $(\tilde{Z}, \tilde{W})$ on the phase space such that the underlying almost-complex structure is given by an elliptic Monge-Ampère operator as above? We shall show that the answer places a restriction on the choice of parameters of the model ( $a$ and $c$ in expression (3.1)), which are defined by the asymptotic regime (balanced approximation to (2.4)) to be modelled.

For an elliptic Monge-Ampère operator with real coefficients,

$$
\omega=\alpha \mathrm{d} p \wedge \mathrm{d} y+\beta(\mathrm{d} q \wedge \mathrm{d} y-\mathrm{d} p \wedge \mathrm{d} x)+\gamma \mathrm{d} x \wedge \mathrm{d} q+\delta \mathrm{d} x \wedge \mathrm{d} y+\epsilon \mathrm{d} p \wedge \mathrm{d} q,
$$

the Pfaffian

$$
\operatorname{Pf}(\omega)=\frac{\omega \wedge \omega}{\Omega \wedge \Omega}=\alpha \gamma-\beta^{2}-\delta \epsilon>0 .
$$

The coordinates $(\tilde{Z}, \tilde{W})$ can be expressed using the coefficients of (4.7):

$$
\tilde{Z}=\alpha_{1} x+\beta_{11} p+\beta_{12} q, \quad \tilde{W}=\alpha_{2} y+\beta_{21} p+\beta_{22} q,
$$

where

$$
\beta_{11}=\alpha, \quad \beta_{22}=\gamma, \quad \beta_{12}=\beta+\mathrm{i}, \quad \beta_{21}=\beta-\mathrm{i}, \quad \alpha_{1}=\alpha_{2}=\delta .
$$

We compute

$$
\operatorname{det} \mathcal{B}=\left|\begin{array}{ll}
\beta_{11} & \beta_{12} \\
\beta_{21} & \beta_{22}
\end{array}\right|=\alpha \gamma-\left(\beta^{2}+1\right),
$$

and, comparing with (4.7), we obtain $\operatorname{det} \mathcal{B}=\epsilon$. Then, using a conformal rescaling by the Pfaffian, we can suppose that, up to a symplectomorphism of $\mathbb{R}^{4}$, either $\delta$ or $\epsilon$ can be chosen to be equal to 1 . It is then easy to see, from (4.6), that in the choice we have for the coordinates $\tilde{Z}, \tilde{W}$, after imposing ellipticity and the conformal choice of $\epsilon=1$, there remain only two degrees of freedom: the dependences on $\delta$ and $\alpha$ (or on $\delta$ and $\gamma$ ).

\section{(c) Conformal structure, pseudometric and special Kähler structure}

Given a pair of 2 -forms $(\omega, \Omega)$ on $T^{*} \mathcal{M}$, such that $\omega \wedge \Omega=0$, then, fixing the volume form in terms of $\Omega$, we can define a conformal pseudo-Riemannian structure on $T^{*} \mathcal{M}$ such that if the volume of $\mathcal{M}$ is $\mathrm{vol}_{2}$, then we have a quadratic form

$$
g_{\omega}(U, V)=\frac{\left(\imath_{U} \Omega \wedge \imath_{V} \omega+\imath_{V} \Omega \wedge \imath_{U} \omega\right)}{\Omega \wedge \Omega} \wedge \pi^{*}\left(\operatorname{vol}_{2}\right),
$$

where $U, V \in T\left(T^{*} \mathcal{M}\right)$ and $\pi: T^{*} \mathcal{M} \mapsto \mathcal{M}$. This fact is relevant to the existence of a conformal $(3,3)$-structure on the Grassmannian $G_{r}(2 ; 4)$.

With an appropriate choice of coordinates, as in (4.7), we have the quadric on $T^{*} \mathcal{M}$ :

$$
g_{\omega}=\gamma x^{2}-2 \beta x y+\alpha y^{2}+\epsilon(p x+q y), \quad g_{\omega} \in \mathcal{S}^{2}\left(T^{*} \mathcal{M}\right) .
$$

For example, if $\omega$ is an elliptic Monge-Ampère operator as in (3.3), $\omega=\hat{\omega}_{I}$, then

$$
g_{\hat{\omega}_{I}}=c^{2}(p x+q y) .
$$

The signature (and, of course, the non-degeneracy) of $g_{\omega}$ is dependent on $\omega$. But if it is non-degenerate (as in the example above) it has signature $(2,2)$. 
A utility of this pseudo-Riemannian structure is that it enables us to unify the elliptic and hyperbolic Monge-Ampère operators in the following sense: we can associate with any Monge-Ampère operator $\omega$ a 'metrically dual' operator $\omega_{g}=$ $g_{\omega} A_{\omega}$, where $A_{\omega}: T\left(T^{*} \mathcal{M}\right) \mapsto T\left(T^{*} \mathcal{M}\right)$ is the field of endomorphisms such that $\omega=\Omega \circ A_{\omega}$. It is a straightforward exercise to show that we have a conformally Kleinian (pseudo-quaternionic) structure on $T^{*} \mathcal{M}$ given by the triple of endomorphisms $\left(A_{\omega}, A_{\omega_{g}}, A_{\omega} \circ A_{\omega_{g}}\right)$. The standard Kleinian structure is given by the triple

$$
\begin{aligned}
\left(A_{\omega^{1}}, A_{\omega_{g}^{1}}, A_{\omega^{1}} \circ A_{\omega_{g}^{1}}\right) & =\left(A_{\omega_{I}^{1}}, A_{g_{I}}, A_{\omega_{I}^{1}} \circ A_{g_{I}}\right) \\
& =\left(I, R, T \mid I^{2}=-1, R^{2}=T^{2}=1, R T I=1\right),
\end{aligned}
$$

and comes from the 2-forms $\omega^{1}=\mathrm{d} x \wedge \mathrm{d} y-\mathrm{d} p \wedge \mathrm{d} q$ and $\omega_{g}^{1}=\mathrm{d} x \wedge \mathrm{d} y+\mathrm{d} p \wedge \mathrm{d} q$. Then, in general,

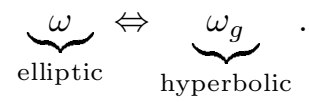

Now, using this pseudo-Riemannian structure, we obtain a non-trivial special Kähler structure on $\mathbb{C}^{2}$ by introducing a holomorphic function $\mathcal{H} \in \mathcal{S}^{2}\left(T^{*} \mathcal{M}\right) \otimes \mathbb{C}$,

$$
\mathcal{H}=S+\tilde{g}_{\omega},
$$

where $S$ is defined in $(4.1)$ and $\tilde{g}_{\omega} \in \mathcal{S}^{2}\left(T^{*} \mathcal{M}\right) \otimes \mathbb{C}$ with

$$
\tilde{g}_{\omega}=\frac{a}{2\left(a^{2}+c^{2}\right)} x^{2}-\frac{\mathrm{i} c}{a^{2}+c^{2}} x y+\frac{a}{2\left(a^{2}+c^{2}\right)} y^{2}+p x+q y .
$$

Hence, the 2-form, associated with this quadric,

$$
\tilde{\omega}=\mathrm{d} p \wedge \mathrm{d} q+\frac{a}{4\left(a^{2}+c^{2}\right)}(\mathrm{d} p \wedge \mathrm{d} y-\mathrm{d} x \wedge \mathrm{d} q)+\frac{\mathrm{i} c}{4\left(a^{2}+c^{2}\right)}(\mathrm{d} q \wedge \mathrm{d} y-\mathrm{d} p \wedge \mathrm{d} x),
$$

defines two real effective forms (i.e. two Monge-Ampère operators)

$$
\begin{aligned}
& \operatorname{Re} \tilde{\omega}=\mathrm{d} p \wedge \mathrm{d} q+\frac{a}{4\left(a^{2}+c^{2}\right)}(\mathrm{d} p \wedge \mathrm{d} y-\mathrm{d} x \wedge \mathrm{d} q), \\
& \operatorname{Im} \tilde{\omega}=\frac{c}{4\left(a^{2}+c^{2}\right)}(\mathrm{d} q \wedge \mathrm{d} y-\mathrm{d} p \wedge \mathrm{d} x),
\end{aligned}
$$

which are hyperbolic:

$$
\begin{aligned}
& \operatorname{Pf}(\operatorname{Re} \tilde{\omega})=-\frac{a^{2}}{16\left(a^{2}+c^{2}\right)}, \\
& \operatorname{Pf}(\operatorname{Im} \tilde{\omega})=-\frac{c^{2}}{16\left(a^{2}+c^{2}\right)} .
\end{aligned}
$$

It is a straightforward calculation to show that

$$
\frac{\partial \mathcal{H}}{\partial Z}=p+\frac{a x}{a^{2}+c^{2}}-\frac{\mathrm{i} c y}{a^{2}+c^{2}}, \quad \frac{\partial \mathcal{H}}{\partial W}=q+\frac{a y}{a^{2}+c^{2}}-\frac{\mathrm{i} c x}{a^{2}+c^{2}},
$$

and

$$
\frac{\partial \mathcal{H}}{\partial \bar{Z}}=0, \quad \frac{\partial \mathcal{H}}{\partial \bar{W}}=0 .
$$


Following Freed (1999), equation (4.18) defines the conjugate coordinates

$$
Z^{\prime}=p+\frac{a x}{a^{2}+c^{2}}-\frac{\mathrm{i} c y}{a^{2}+c^{2}}, \quad W^{\prime}=q+\frac{a y}{a^{2}+c^{2}}-\frac{\mathrm{i} c x}{a^{2}+c^{2}},
$$

and the Hessian,

$$
\operatorname{Hess}(\mathcal{H})=\left(\begin{array}{cc}
\frac{\partial^{2} \mathcal{H}}{\partial Z^{2}} & \frac{\partial^{2} \mathcal{H}}{\partial Z \partial W} \\
\frac{\partial^{2} \mathcal{H}}{\partial W \partial Z} & \frac{\partial^{2} \mathcal{H}}{\partial W^{2}}
\end{array}\right)=\left(\begin{array}{cc}
\frac{a}{a^{2}+c^{2}} & -\frac{i c}{a^{2}+c^{2}} \\
-\frac{i c}{a^{2}+c^{2}} & \frac{a}{a^{2}+c^{2}}
\end{array}\right),
$$

satisfies $|\operatorname{Hess}(\mathcal{H})|=1 /\left(a^{2}+c^{2}\right)$. $\mathcal{H}$ is called a holomorphic prepotential of a special Kähler structure. Furthermore, let $\left\|\tau_{i j}\right\|=\|\operatorname{Hess}(\mathcal{H})\|$, then

$$
g_{i j}=\operatorname{Im} \tau_{i j}=\left(\begin{array}{cc}
0 & -\frac{c}{a^{2}+c^{2}} \\
-\frac{c}{a^{2}+c^{2}} & 0
\end{array}\right)
$$

and the function $\mathcal{K}=\frac{1}{2} \operatorname{Im}\left(Z^{\prime} \bar{Z}+W^{\prime} \bar{W}\right)$ is a Kähler potential, in terms of which an associated Kähler form is $\omega^{\prime}=\mathrm{i} \partial \bar{\partial} \mathcal{K}(Z, W)$.

As an example of the foregoing, let us consider the Monge-Ampère operators associated with the transformations (3.1) and (3.16). We have the 2-form

$\mathrm{d} X \wedge \mathrm{d} Y=\mathrm{d} x \wedge \mathrm{d} y+\left(a^{2}-c^{2}\right) \mathrm{d} p \wedge \mathrm{d} q+a(\mathrm{~d} x \wedge \mathrm{d} q+\mathrm{d} p \wedge \mathrm{d} y)-\mathrm{i} c(\mathrm{~d} x \wedge \mathrm{d} p+\mathrm{d} y \wedge \mathrm{d} q)$.

The effective part of this form that corresponds to the Monge-Ampère operator (Lychagin 1979) is the real part, $\operatorname{Re}(\mathrm{d} X \wedge \mathrm{d} Y)$, of $\mathrm{d} X \wedge \mathrm{d} Y$. In our notation (4.7) $\alpha=\gamma=a, \epsilon=a^{2}-c^{2}, \delta=1$. Hence, the Pfaffian $\operatorname{Pf}(\operatorname{Re}(\mathrm{d} X \wedge \mathrm{d} Y))=c^{2}$, and we obtain an elliptic Monge-Ampère operator together with a new (conformal) almost complex structure on $\mathbb{R}^{4}$, which was implicit in the McIntyre \& Roulstone (1996) description of balanced models:

$$
\left.\begin{array}{rlrl}
\frac{\partial}{\partial x} & \mapsto a \frac{\partial}{\partial y}+\frac{\partial}{\partial q}, & \mapsto a \frac{\partial}{\partial x}-\frac{\partial}{\partial p}, \\
\frac{\partial}{\partial p} \mapsto-\left(a^{2}-c^{2}\right) \frac{\partial}{\partial y}-a \frac{\partial}{\partial q}, & \frac{\partial}{\partial q} \mapsto\left(a^{2}-c^{2}\right) \frac{\partial}{\partial x}+a \frac{\partial}{\partial p} .
\end{array}\right\}
$$

This structure is obviously integrable in the constant coefficient case.

We can associate the form $\operatorname{Re}(\mathrm{d} X \wedge \mathrm{d} Y)$ (and the associated Monge-Ampère operator) with its 'dual' counterpart, $\mathcal{J}$, by using the above mentioned (2,2)-pseudo-metric associated with $\operatorname{Re}(\mathrm{d} X \wedge \mathrm{d} Y)$ :

$$
\mathcal{J}=-2 \mathrm{~d} p \wedge \mathrm{d} q+\frac{2\left(a^{2}+c^{2}\right)}{\left(a^{2}-c^{2}\right)^{2}} \mathrm{~d} x \wedge \mathrm{d} y-\frac{2 a}{a^{2}-c^{2}}(\mathrm{~d} x \wedge \mathrm{d} q+\mathrm{d} p \wedge \mathrm{d} y)
$$

with the Pfaffian

$$
\operatorname{Pf}(\mathcal{J})=-\frac{4 c^{2}}{\left(a^{2}-c^{2}\right)^{2}}
$$

It is clear that this 'dual' form exists if and only if $a^{2} \neq c^{2}$. The singular values of the parameters correspond to a degeneration of the pseudo-metric. 
On the other hand, for the 'deformed' coordinates $Z, W$, the analogous calculations give us two hyperbolic Monge-Ampère operators, which correspond to two effective 2 -forms $\omega_{1}, \omega_{2}$, such that

$$
\mathrm{d} Z \wedge \mathrm{d} W=\omega_{1}+\mathrm{i} \omega_{2} .
$$

Their Pfaffians are equal: $\operatorname{Pf}\left(\omega_{1}\right)=\operatorname{Pf}\left(\omega_{2}\right)=-c^{2}$, and hence they define symplectically equivalent Monge-Ampère operators. The choice of the parameters $a=1$, $c=\sqrt{ } 3$ in the McIntyre-Roulstone model implies that the 'dual' hyperbolic operator that corresponds to the form $\mathcal{J}$ is symplectically equivalent to both operators defined by the pair $\omega_{1}, \omega_{2}$. Such 'duality' is the subject of ongoing research.

We should make a further remark about these special Lagrangian coordinates. As discussed by Hitchin (1997) and Gross (1999), the salient feature of their existence is the question of the integrability to the corresponding almost-complex structure. This question looks trivial in the 'linear' approximation we have discussed here, though the theorem of Lie in the form of Lychagin \& Roubtsov (1983) gives the integrability conditions in terms of

$$
\mathrm{d} \omega=\frac{1}{2} \mathrm{~d} \log |\operatorname{Pf}(\omega)| \wedge \omega
$$

for the corresponding effective Monge-Ampère form (another version of this statement is known also as 'Hitchin's lemma' (see Hitchin 1987)). The foregoing special Lagrangian structure has relied on $\omega$, in (4.7), having constant coefficients: in these cases the Ricci-flat condition is trivially satisfied and some interesting cases when this is not so are the subject of a further study.

\section{(d) A contact transformation}

Finally, we show how the contact structure of the semi-geostrophic equations, which is perhaps the most important mathematical property of these equations because it facilitates practical solution strategies (see Purser (1993) and references therein; see also Roulstone \& Sewell (1997) and Sewell \& Roulstone (1994)), generalizes to other near-local balanced models.

We can introduce a 'semi-holomorphic' contact bundle corresponding to the coordinates $(Z, W)$ with local coordinates $(Z, W ; \Phi ; P, Q)$ where, as in (4.1) and those equations that follow, $\Phi=\varphi+S$ and

$$
P=\frac{\partial \Phi}{\partial Z}=p, \quad Q=\frac{\partial \Phi}{\partial W}=q
$$

One can show that

$$
\mathrm{d} \Phi=\mathrm{d} \varphi+\mathrm{d} S=\mathrm{d} \varphi+\mathrm{i} c(p \mathrm{~d} q+q \mathrm{~d} p)+a(p \mathrm{~d} p+q \mathrm{~d} q),
$$

and, furthermore,

$$
\mathrm{d} \Phi-\frac{\partial \Phi}{\partial Z} \mathrm{~d} Z-\frac{\partial \Phi}{\partial W} \mathrm{~d} W=\mathrm{d} \varphi-p \mathrm{~d} x-q \mathrm{~d} y
$$

This is a 'contactification' of the conformal symplectomorphism (3.14). 


\section{Summary}

These results are a contribution to the ongoing research into balanced models in geophysical fluid dynamics, in which the objective is to understand the properties of the equations that assist solution strategies. The relationships between the ellipticity of the operators, the Hamiltonian structure of the evolution equations and the stability of flows described by such balanced models are subtle and our understanding of these issues is far from complete. This paper demonstrates how geometry can offer insights into some of these problems. For example, the Kähler structure associated with a Monge-Ampère equation facilitates an understanding of

(i) why complex coordinates arise in a natural way in these models, something that would be difficult to deduce from the physics alone, and

(ii) how the elliptic and hyperbolic operators are related under duality.

We have shown that a natural generalization (3.1) of the so-called geostrophic momentum transformation (2.8) of semi-geostrophic theory, discovered by McIntyre \& Roulstone (1996), possesses a special Lagrangian and special Kähler structure. The conditions under which these properties exist (a Monge-Ampère equation with constant coefficients) may appear a little restrictive from the point of view of the hydrodynamics, but the identification of these geometries has, in turn, facilitated an approach to studying the duality structure of the models as described in $\S 4 c$ and $\S 4 d$. A study with less restrictive integrability conditions is in progress. The duality properties of semi-geostrophic theory have been studied extensively elsewhere (see, for example, Roulstone \& Sewell (1997) and references cited therein), and such features are of practical importance too, noting both the utility of balanced dynamics in understanding mid-latitude weather systems and the attempts to improve the representation of such weather systems in numerical models via, for example, the use of adaptive techniques in data-assimilation schemes (see, for example, Desroziers 1997).

The authors are grateful to Professors N. J. Hitchin and M. E. McIntyre for useful discussions. The work was supported by the European Science Exchange Programme Joint Project Scheme under cooperation between The Royal Society and the Centre National de la Recherche Scientifique. V.N.R. was partly supported by grant RFFI-98-01-00327 and by grant 00-15-9650057 for scientific schools. This paper is copyright of the Controller, Her Majesty's Stationery Office, Norwich, England, 2001.

\section{References}

Allen, J. S., Barth, J. A. \& Newberger, P. A. 1990 On intermediate models for barotropic continental shelf and slope flow fields. Part I. Formulation and comparison of exact solutions. J. Phys. Ocean. 20, 1017-1042.

Chynoweth, S. \& Sewell, M. J. 1989 Dual variables in semi-geostrophic theory. Proc. R. Soc. Lond. A 424, 155-186.

Chynoweth, S. \& Sewell, M. J. 1991 A concise derivation of the semi-geostrophic equations. $Q$. J. R. Meteorol. Soc. 117, 1109-1128.

Cullen, M. J. P. \& Purser, R. J. 1984 An extended Lagrangian theory of semi-geostrophic frontogenesis. J. Atmos. Sci. 41, 1477-1497.

Cullen, M. J. P., Norbury, J. \& Purser, R. J. 1991 Generalised Lagrangian solutions for atmospheric and oceanic flows. SIAM J. Appl. Math. 51, 20-31.

Proc. R. Soc. Lond. A (2001) 
Desroziers, G. 1997 A coordinate change for data assimilation in spherical geometry of frontal structures. Mon. Wea. Rev. 125, 3030-3038.

Freed, D. S. 1999 Special Kähler manifolds. Commun. Math. Phys. 203, 31-52.

Gross, M. 1999 Special Lagrangian fibrations. II. Geometry. Surv. Diff. Geom. 5, 341-403.

Hitchin, N. J. 1987 Monopoles, minimal surfaces and algebraic curves. In NATO Advanced Study Institute, vol. 105, L'Université de Montréal.

Hitchin, N. J. 1997 The moduli space of special Lagrangian submanifolds. Ann. Scu. Ec. Norm. Super. Pisa 25, 503-515.

Hoskins, B. J. 1975 The geostrophic momentum approximation and the semi-geostrophic equations. J. Atmos. Sci. 32, 233-242.

Lychagin, V. V. 1979 Non-linear differential equations and contact geometry. Russ. Math. Surv. 34, 137-165.

Lychagin, V. V. \& Roubtsov, V. N. 1983 On Sophus Lie theorems for Monge-Ampère equations. (In Russian.) Doklady Bielorussian Academy Sci. 27, 396-398.

Lychagin, V. V., Roubtsov, V. N. \& Chekalov, I. V. 1993 A classification of Monge-Ampère equations. Ann. Sci. Ec. Norm. Super. 26, 281-308.

McIntyre, M. E. \& Roulstone, I. 1996 Hamiltonian balanced models: constraints, slow manifolds and velocity splitting. Forecasting Research Scientific Paper, no. 41, Met Office, UK. Corrected version in preparation. Full text and corrections are available at http://www.atm. damtp.cam.ac.uk/people/mem/.

McIntyre, M. E. \& Roulstone, I. 2001 Are there higher-accuracy analogues of semi-geostrophic theory? In Large scale atmosphere-ocean dynamics, vol. II, Geometric methods and models (ed. I. Roulstone \& J. Norbury). Cambridge University Press. (In the press.)

McWilliams, J. C. \& Gent, P. R. 1980 Intermediate models of planetary circulations in the atmosphere and ocean. J. Atmos. Sci. 37, 1657-1678.

Purser, R. J. 1993 Contact transformations and Hamiltonian dynamics in generalized semigeostrophic theories. J. Atmos. Sci. 50, 1449-1468.

Purser, R. J. \& Cullen, M. J. P. 1987 A duality principle in semi-geostrophic theory. J. Atmos. Sci. 44, 3449-3468.

Roubtsov, V. N. \& Roulstone, I. 1997 Examples of quaternionic and Kähler structures in Hamiltonian models of nearly geostrophic flow. J. Phys. A 30, L63-L68.

Roulstone, I. \& Norbury, J. 1994 A Hamiltonian structure with contact geometry for the semigeostrophic equations. J. Fluid Mech. 272, 211-233.

Roulstone, I. \& Sewell, M. J. 1997 The mathematical structure of theories of semi-geostrophic type. Phil. Trans. R. Soc. Lond. A 355, 2489-2517.

Salmon, R. 1983 Practical use of Hamilton's principle. J. Fluid Mech. 132, 431-444.

Salmon, R. 1985 New equations for nearly geostrophic flow. J. Fluid Mech. 153, 461-477.

Salmon, R. 1988 Semi-geostrophic theory as a Dirac bracket projection. J. Fluid Mech. 196, 345-358.

Sewell, M. J. \& Roulstone, I. 1994 Families of lift and contact transformations. Proc. R. Soc. Lond. A 447, 493-512. 